\title{
Role of the electronic structure and multielectron responses in ionization mechanisms of diatomic molecules in intense short-pulse lasers: An all-electron ab initio study
}

\author{
$\mathrm{Xi} \mathrm{Chu}$ \\ ITAMP, Harvard-Smithsonian Center for Astrophysics, 60 Garden Street, Cambridge, Massachusetts 02138, USA
}

Shih-I Chu

Department of Chemistry, University of Kansas, and Kansas Center for Advanced Scientific Computing, Lawrence, Kansas 66045, USA

(Received 6 July 2004; revised manuscript received 12 October 2004; published 14 December 2004)

\begin{abstract}
We present an all-electron ab initio study of multiphoton ionization (MPI) of diatomic molecules in intense laser pulses using the example of $\mathrm{N}_{2}, \mathrm{O}_{2}$, and $\mathrm{F}_{2}$, and the theoretical approach of time-dependent densityfunctional theory with correct long-range potential. The results reveal the importance of the electronic structure and correlated multielectron responses in the ionization mechanism, and make evident inner valence electron contributions to the molecular MPI in strong laser fields.
\end{abstract}

DOI: 10.1103/PhysRevA.70.061402

The single electron ionization of molecules in ultrashort intense laser fields is a key process leading to a number of strong-field phenomena, such as above-threshold ionization, multiphoton ionization and dissociation, high harmonic generation, Coulomb explosion, nonsequential multielectron ionization, etc. Thus a detailed understanding of the ionization mechanism is a prerequisite for the exploration of molecular physics in strong fields.

The strong-field ionization of rare-gas atoms has been well studied by solving time-dependent Schrödinger equations with the single active electron (SAE) approximation [1-3]. In the tunneling regime [4], the Ammosov-DeloneKrainov (ADK) model [5] (based on SAE and other approximations) has also been used to study the single and sequential multielectron ionization of rare-gas atoms. In the ADK model, the response of an atom depends only upon the ionization potential (IP) of the outermost atomic orbital, and details of the electronic structure are not considered.

Multiphoton ionization of molecules, however, is considerably more complicated and the understanding of its mechanisms remains unsettled. Earlier experimental studies [6-8] suggested that ionization rates of molecules are similar to noble gas atoms with similar IP, although more recent experiments have found exceptions [9-12]. The ionization is suppressed for $\mathrm{O}_{2}$, in comparison with $\mathrm{Xe}$, while the ionization of $\mathrm{N}_{2}$ and $\mathrm{F}_{2}$ are comparable to their comparison atom Ar. Without an $a b$ initio study of time-dependent dynamics of many-electron molecules, recent theoretical studies of molecular ionization behavior have relied upon approximate models such as the ADK [13], KFR (or Keldysh-FaisalReiss) [14,15], or screening [16] models. The screening model [16] introduces a charge-screening correction to the tunneling theory. The KFR model [15] predicts that the interference between electrons emitted from the vicinity of two distinct ionic centers can lead to ionization suppression for molecules with antisymmetric electronic ground states. All three models correctly predict the suppressed ionization of $\mathrm{O}_{2}$, and the absence of suppression of $\mathrm{N}_{2}$. However, the ADK and KFR models also predict the ionization suppression of $F_{2}[13,15]$, which is in disagreement with recent experimental results [9-12].
We present in this article a three-dimensional all-electron nonperturbative investigation of mechanisms of the general strong-field ionization behavior, using $\mathrm{N}_{2}, \mathrm{O}_{2}$, and $\mathrm{F}_{2}$ as examples, and taking into account the detailed electronic structure and responses of individual electrons. Groundstate electronic configurations of $\mathrm{N}_{2}, \mathrm{O}_{2}$, and $\mathrm{F}_{2}$ are $\quad K K 2 \sigma_{g}^{2} 2 \sigma_{u}^{2} 1 \pi_{u}^{4} 3 \sigma_{g}^{2}, \quad K K 2 \sigma_{g}^{2} 2 \sigma_{u}^{2} 3 \sigma_{g}^{2} 1 \pi_{u}^{4} 1 \pi_{g}^{2}, \quad$ and $K K 2 \sigma_{g}^{2} \sigma_{u}^{2} 3 \sigma_{g}^{2} 1 \pi_{u}^{4} 1 \pi_{g}^{4}$, respectively. We obtain the initial electronic state by solving static density-functional theory (DFT) equations with $\mathrm{LB}_{\alpha}[17,18]$ potential,

$$
\begin{aligned}
v_{x c \sigma}^{L B \alpha}(\mathbf{r}, t)= & \alpha v_{x \sigma}^{L S D A}(\mathbf{r}, t)+v_{c \sigma}^{L S D A}(\mathbf{r}, t) \\
& -\frac{\beta x_{\sigma}^{2}(\mathbf{r}, t) \rho_{\sigma}^{1 / 3}(\mathbf{r}, t)}{1+3 \beta x_{\sigma}(\mathbf{r}, t) \ln \left\{x_{\sigma}(\mathbf{r}, t)+\left[x_{\sigma}^{2}(\mathbf{r}, t)+1\right]^{1 / 2}\right\}},
\end{aligned}
$$

where the first two terms are the LSDA exchange and correlation potentials, and the last term is the gradient correction that ensures $v_{x c \sigma}^{L B \alpha} \rightarrow-1 / r$ as $r \rightarrow \infty$, and it produces accurate excited states as well as the ground state. For $\mathrm{N}_{2}$ and $\mathrm{O}_{2}$ we choose $\alpha=1.19$ and $\beta=0.01$; for $\mathrm{F}_{2}$ we let $\alpha=1.16$ and $\beta$ $=0.01$. We use the generalized pseudospectral (GPS) method for two-center systems [19], which features nonuniform and optimal spatial grids and achieves machine accuracy numerical convergence with the minimum number of grid points [19]. Table I compares the calculated orbital binding energies $(-\epsilon)$ with measured vertical ionization energies, where internuclear distances are fixed at the equilibrium $R_{e}$ of the ground state of neutral molecules. Calculated and measured values of $3 \sigma_{g}, 1 \pi_{u}$, and $2 \sigma_{u}$ orbital energies of $\mathrm{N}_{2}$ agree excellently. Other orbitals have large binding energies and do not participate in the ionization processes we study. After the removal of an electron the remaining $\mathrm{O}_{2}{ }^{+}$ion has two multiplets. Differences between the calculated and measured binding energies of the three $\Pi$ states are within $0.13 \mathrm{eV}$. The calculated $1 \pi_{g}$ and $1 \pi_{u}$ orbital energies of $\mathrm{F}_{2}$ agree well with experimental values.

We further solve a set of time-dependent equations,

$$
\begin{aligned}
i \frac{\partial}{\partial t} \psi_{i \sigma}(\mathbf{r}, t) & =\hat{H}(\mathbf{r}, t) \psi_{i \sigma}(\mathbf{r}, t) \\
& =\left[-\frac{1}{2} \nabla^{2}+V_{e f f, \sigma}([\rho] ; \mathbf{r}, t)\right] \psi_{i \sigma}(\mathbf{r}, t),
\end{aligned}
$$


TABLE I. Comparison of calculated binding energies of diatomic molecules and experimental values.

\begin{tabular}{|c|c|c|c|c|c|}
\hline Molecule & $\begin{array}{c}\text { Bond } \\
\text { length }\left(a_{0}\right)\end{array}$ & $\begin{array}{l}\text { Orbital } \\
\text { ionized }\end{array}$ & $\begin{array}{l}\text { Ion } \\
\text { state }\end{array}$ & $\begin{array}{c}-\epsilon \\
(\mathrm{eV})\end{array}$ & $\begin{array}{c}I_{\text {vert }} \\
(\mathrm{eV})[23-25]\end{array}$ \\
\hline \multirow[t]{4}{*}{$\mathrm{N}_{2}$} & \multirow{4}{*}{$\begin{array}{c}2.068 \\
{[23]}\end{array}$} & $2 \sigma_{g}$ & ${ }^{2} \Sigma_{g}^{+}$ & 33.09 & 37.3 \\
\hline & & $2 \sigma_{u}$ & ${ }^{2} \Sigma_{u}^{+}$ & 18.48 & 18.6 \\
\hline & & $1 \pi_{u}$ & ${ }^{2} \Pi_{u}^{u}$ & 16.87 & 16.8 \\
\hline & & $3 \sigma_{g}$ & ${ }^{2} \Sigma_{g}^{+}$ & 15.49 & 15.5 \\
\hline \multirow[t]{7}{*}{$\mathrm{O}_{2}$} & \multirow{7}{*}{$\begin{array}{c}2.287 \\
{[24]}\end{array}$} & \multirow[t]{2}{*}{$2 \sigma_{u}$} & ${ }^{2} \Sigma_{u}^{-}$ & 25.16 & 27.3 \\
\hline & & & ${ }^{4} \Sigma_{u}^{-}$ & 23.41 & 24.56 \\
\hline & & \multirow[t]{2}{*}{$3 \sigma_{g}$} & ${ }^{2} \Sigma_{g}^{-}$ & 18.64 & 20.30 \\
\hline & & & ${ }^{4} \Sigma_{g}^{-}$ & 18.56 & 18.17 \\
\hline & & \multirow[t]{2}{*}{$1 \pi_{u}$} & ${ }^{2} \Pi_{u}^{8}$ & 17.56 & 17.64 \\
\hline & & & ${ }^{4} \Pi_{u}$ & 16.82 & 16.70 \\
\hline & & $1 \pi_{g}$ & ${ }^{2} \Pi_{g}$ & 11.93 & 12.06 \\
\hline \multirow[t]{4}{*}{$\mathrm{F}_{2}$} & \multirow{4}{*}{$\begin{array}{c}2.665 \\
{[25]}\end{array}$} & $2 \sigma_{u}$ & ${ }^{2} \Sigma_{u}^{+}$ & 33.83 & \\
\hline & & $3 \sigma_{g}$ & ${ }^{2} \Sigma_{g}^{+}$ & 21.61 & 21 \\
\hline & & $1 \pi_{u}$ & ${ }^{2} \Pi_{u}^{s}$ & 18.98 & 18.98 \\
\hline & & $1 \pi_{g}$ & ${ }^{2} \Pi_{g}$ & 15.70 & 15.70 \\
\hline
\end{tabular}

$$
i=1,2, \ldots, N_{\sigma},
$$

where $\left\{\psi_{i \sigma}\right\}$ is the set of occupied single-electron orbital wave functions at time $t$, and

$V_{e f f, \sigma}([\rho] ; \mathbf{r}, t)=v_{e x t}(\mathbf{r}, t)+\int \frac{\rho\left(\mathbf{r}^{\prime}, t\right)}{\left|\mathbf{r}-\mathbf{r}^{\prime}\right|} d^{3} \mathbf{r}^{\prime}+v_{x c, \sigma}(\mathbf{r}, t)$,

where $v_{\text {ext }}(\mathbf{r}, t)$ is the "external" potential due to the interaction of the electron with the external laser field and the nuclei. For a homonuclear molecule in a linearly polarized external laser field, we have

$$
v_{\text {ext }}(\mathbf{r}, t)=-\frac{Z}{\left|\mathbf{R}_{1}-\mathbf{r}\right|}-\frac{Z}{\left|\mathbf{R}_{2}-\mathbf{r}\right|}+\mathbf{E}(t) \cdot \mathbf{r} \sin \omega t,
$$

in which $\mathbf{r}$ is the electronic coordinate, $\mathbf{E}(t)$ the electric-field amplitude, $\mathbf{R}_{1}$ and $\mathbf{R}_{2}$ are the coordinates of the two nuclei, and $Z$ is the nuclear charge. The internuclear separation is fixed at the equilibrium. $v_{x c \sigma}(\mathbf{r}, t)$ is the time-dependent exchange-correlation (xc) potential.

We solve Eq. (1) by the TDGPS method [20]. The timedependent ionization probability of an electron in the $i$, $\sigma$ th spin-orbital can be calculated according to $P_{i, \sigma}=1-N_{i, \sigma}(t)$, where $N_{i \sigma}(t)=\left\langle\psi_{i, \sigma}(t) \mid \psi_{i, \sigma}(t)\right\rangle$ is the time-dependent electron population of the $i$, $\sigma$ th spin-orbital. The total ionization probability $P$ can be obtained from the sum $P=\Sigma_{i, \sigma} P_{i, \sigma}$.

A plot of $N_{i \sigma}(t)$ against the time allows us to observe each electron's contribution to the total ionization probability, ionization rate, and how they relate to one another. We use 800-nm, 20-optical-cycle lasers as an example; there are no obvious resonances at this frequency. Three intensities are applied to each molecule to identify the effects of laser intensity on the ionization mechanism. The nuclei are fixed at the equilibrium distances because pulse lengths of the lasers are much shorter than the vibration relaxation time of the
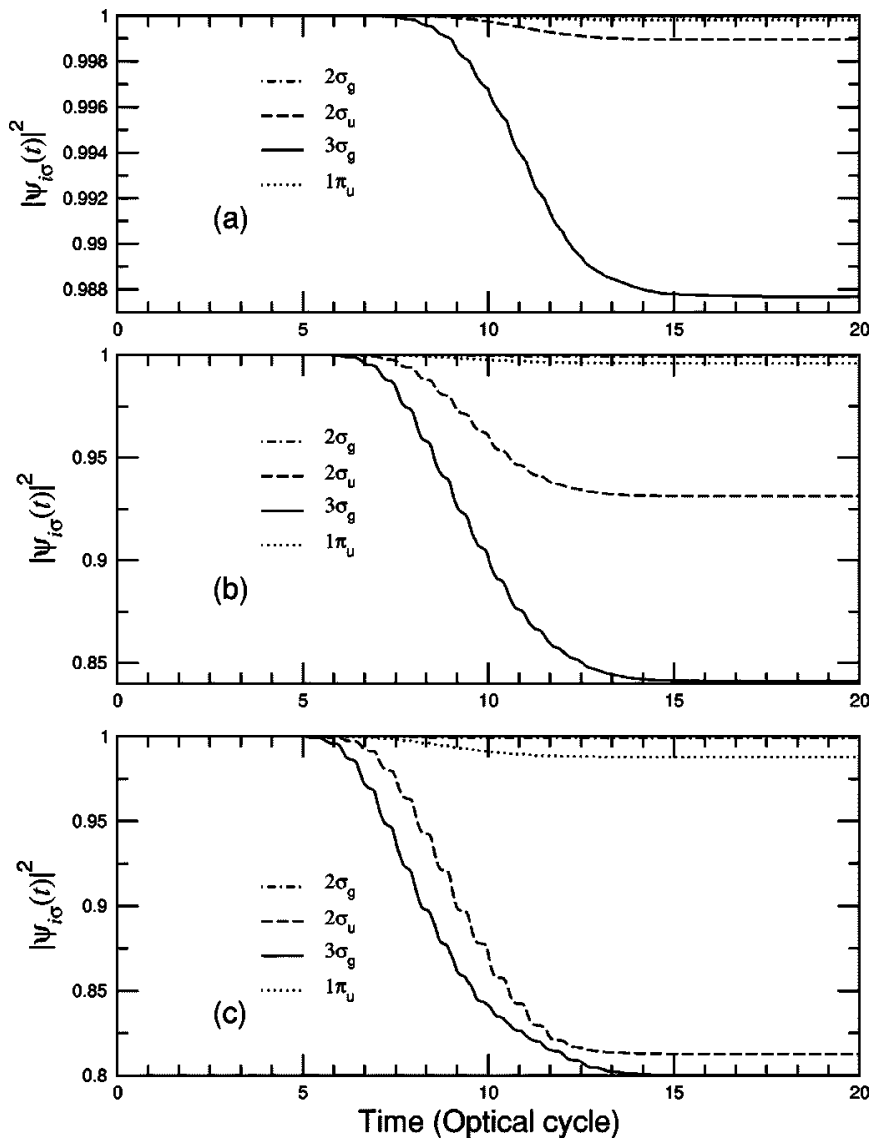

FIG. 1. The time-dependent electron population of individual spin-orbitals of $\mathrm{N}_{2}$ at $R=2.068 a_{0}$ in 20-optical-cycle, 800- nm, $\sin ^{2}$ laser pulses. The laser intensities are (a) $10^{14}$, (b) $3 \times 10^{14}$, and (c) $5 \times 10^{14} \mathrm{~W} / \mathrm{cm}^{2}$.

molecules. We make the molecular axis parallel to the polarization direction of the laser to look at the orbital orientation effect at one particular angle. In Figs. 1-3 we present such plots for $\mathrm{N}_{2}, \mathrm{O}_{2}$, and $\mathrm{F}_{2}$, respectively, and the curves are labeled by the initial orbitals for $\mathrm{N}_{2}$ and $\mathrm{F}_{2}$, and by the ion states for $\mathrm{O}_{2}$.

Figure 1 demonstrates how $\mathrm{N}_{2}$ ionizes when the laser intensity varies from $10^{14} \mathrm{~W} / \mathrm{cm}^{2}$ (a) to $5 \times 10^{14} \mathrm{~W} / \mathrm{cm}^{2}$ (c). The total ionization probability increases from 0.0285 [Fig. 1(a)] to 0.474 [Fig. 1(b)] and 0.825 [Fig. 1(c)]; the probability for the photoelectron being a $2 \sigma_{u}$ electron increases from $7.4 \%$ (a) to $29.1 \%$ (b), and $48.4 \%$ (c). It indicates that around the saturation intensity, the photoelectron has a probability distribution of electrons of different binding energies; high laser intensities tend to enhance the dipole coupling of $3 \sigma_{g}$ and $2 \sigma_{u}$ orbitals. The ionization of $1 \pi_{u}$ electrons is not evident when the laser field is parallel to the molecular axis, although the IP of $1 \pi_{u}$ electrons is $2-\mathrm{eV}$ less than that of the $2 \sigma_{u}$ electrons.

We apply the same lasers to $\mathrm{O}_{2}$ (Fig. 2). The binding energy of the highest electron is 3.56-eV less than that of $\mathrm{N}_{2}$; it is an open-shell molecule, and the two $1 \pi_{g}$ orbitals can only couple to the like-spin orbitals. The total ionization probability of Figs. 2(b) and 2(c) is smaller than that of Figs. 1(b) and 1(c), respectively; a $1 \pi_{g}$ electron in Fig. 2(c) has a smaller ionization probability than a $3 \sigma_{g}$ or a $2 \pi_{u}$ electron in 

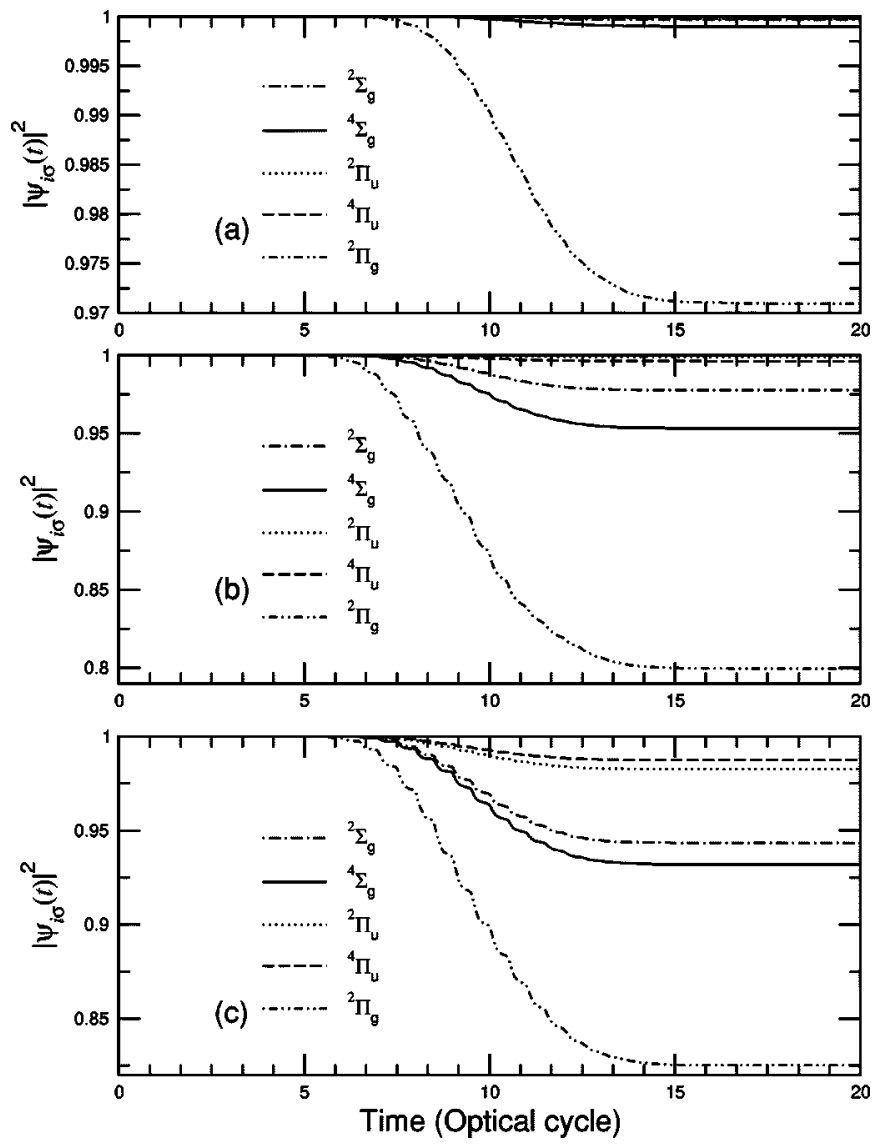

FIG. 2. The time-dependent electron population of individual spin-orbitals of $\mathrm{O}_{2}$ at $R=2.287 a_{0}$ in 20-optical-cycle, 800-nm, $\sin ^{2}$ pulses. The curves are labeled with the ion state upon the removal of an electron. The laser intensities are (a) $10^{14}$, (b) $3 \times 10^{14}$, and (c) $5 \times 10^{14} \mathrm{~W} / \mathrm{cm}^{2}$.

Fig. 1(c), which proves that the binding energy is not the only parameter that determines the ionization probability. We also notice that the photoelectron generated by the MPI of $\mathrm{O}_{2}$ is more likely to be the highest electron. Figure 2 can also help to examine the relationship between ionization suppression of $\mathrm{O}_{2}$ and multielectron responses. With the 3 $\times 10^{14} \mathrm{~W} / \mathrm{cm}^{2}$ pulse, the total ionization probability is 0.482 , and $83.1 \%$ of the ionization is from $1 \pi_{g}$ electrons. When the laser intensity reaches $5 \times 10^{14} \mathrm{~W} / \mathrm{cm}^{2}$, as in Fig. 2 (c), the total ionization probability becomes 0.537 , and $65.1 \%$ of the ionization is from $1 \pi_{g}$. The ionization probability of a $1 \pi_{g}$ electron decreases from 0.2 to 0.175 , which means that the multielectron responses suppress the ionization of the highest electrons of $\mathrm{O}_{2}$. The MPI of $1 \pi_{u}$ electrons is not significant, which is because $\mathrm{O}_{2}$ does not have as strong a coupling between $1 \pi_{g}$ and $1 \pi_{u}$ orbitals as that between the $3 \sigma_{g}$ and $2 \sigma_{u}$ orbitals of $\mathrm{N}_{2}$. The $3 \sigma_{g}$ ionization is evident because of the orbital orientation.

$\mathrm{F}_{2}$ is similar to $\mathrm{N}_{2}$ as a closed-shell molecule, and the energy separation of the $1 \pi_{g}$ and $1 \pi_{u}$ orbitals of $\mathrm{F}_{2}$ is close to the energy separation of the $2 \sigma_{u}$ and $3 \sigma_{g}$ orbitals of $\mathrm{N}_{2}$; it is also similar to $\mathrm{O}_{2}$ because it has the same type of molecular orbitals. The total ionization probabilities in Figs. 3(a)-3(c) are $0.00152,0.372$, and 0.697, respectively. As the intensity increases, the ionization probabilities of a $1 \pi_{g}$ and a
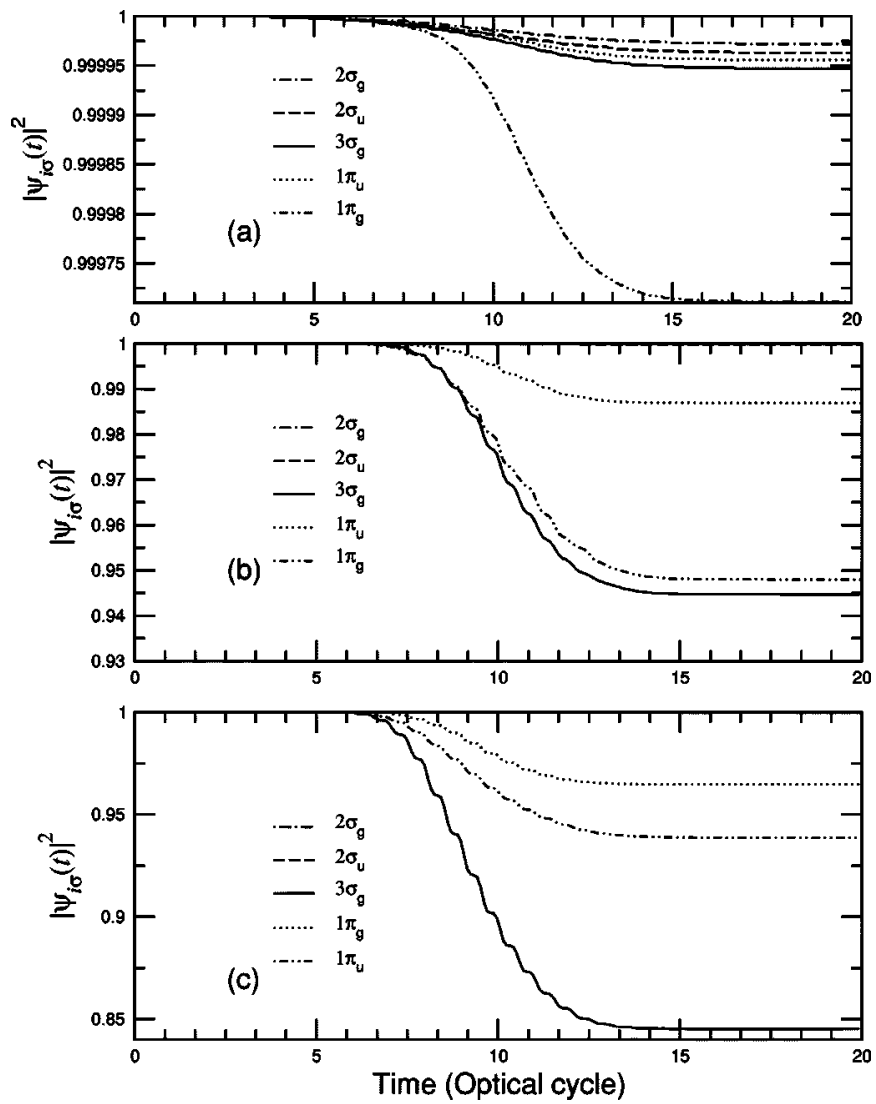

FIG. 3. The time-dependent electron population of individual spin-orbitals of $\mathrm{F}_{2}$ at $R=2.665 a_{0}$ in 20-optical-cycle, 800-nm, $\sin ^{2}$ laser pulses. The laser intensities are (a) $10^{14}$, (b) $3 \times 10^{14}$, and (c) $5 \times 10^{14} \mathrm{~W} / \mathrm{cm}^{2}$.

$1 \pi_{u}$ electron get closer, and $3 \sigma_{g}$ electrons become the most ionized electrons. The increasing percentage of the $1 \pi_{u}$ ionization from Figs. 3(a)-3(c) is due to the enhancement of the $1 \pi_{g}$ and $1 \pi_{u}$ coupling with increasing laser intensities.

We summarize in Table II the total ionization probabilities that correspond to Figs. $1-3$. At 3 and $5 \times 10^{14} \mathrm{~W} / \mathrm{cm}^{2}$, the ratio of the ionization probability of $\mathrm{N}_{2}$ to $\mathrm{F}_{2}$ is 1.3 , which is consistent with the experimental data presented in Fig. 2 of [11] using 790-nm lasers of a longer pulse duration. Direct numerical comparisons with $\mathrm{O}_{2}$ are not as informative because of the differences in the ionization potential. Nevertheless, that $\mathrm{N}_{2}, \mathrm{O}_{2}$, and $\mathrm{F}_{2}$ have similar total ionization probabilities at higher intensities indicates both the ionization suppression for $\mathrm{O}_{2}$ and the nonsuppression for $\mathrm{F}_{2}$. At $10^{14} \mathrm{~W} / \mathrm{cm}^{2}$, the ionization probability of $\mathrm{N}_{2}$ is 19 times that of $\mathrm{F}_{2}$, when the molecular axis is parallel to the polarization direction of the laser. Figure 2 of [11] indicates nearly identical values when all molecular orientations are taken into account. The difference is due to the orientation effect that maximizes the ionization of the $3 \sigma_{g}$ electrons of $\mathrm{N}_{2}$ [21] and minimizes that of the $1 \pi_{g}$ electrons of $\mathrm{F}_{2}$ [13] at the parallel orientation. Our calculations suggest that the anisotropy of the ionization of $\mathrm{N}_{2}$ or $\mathrm{F}_{2}$ is more significant at less laser intensities, where only one type of electrons, the highest electrons, are most active. Whereas at higher intensities, different inner valence electrons corresponding to different molecular orientations ionize significantly and reduce the anisotropy of the total ionization probability [22]. 
TABLE II. Ionization probabilities of $\mathrm{N}_{2}, \mathrm{O}_{2}$, and $\mathrm{F}_{2}$, and the contributions from different spin-orbitals.

\begin{tabular}{ccccccc}
\hline \hline $\begin{array}{c}\text { Laser intensity } \\
\left(\mathrm{W} / \mathrm{cm}^{2}\right)\end{array}$ & Molecule & ionization probability & $2 \sigma_{u}$ & $3 \sigma_{g}$ & $1 \pi_{u}$ & $1 \pi_{g}$ \\
\hline \multirow{2}{*}{$10^{14}$} & $\mathrm{~N}_{2}$ & 0.0285 & 0.0021 & 0.0248 & 0.0012 & \\
& $\mathrm{O}_{2}$ & 0.0601 & 0.0002 & 0.0013 & 0.0004 & 0.0582 \\
& $\mathrm{~F}_{2}$ & 0.00152 & 0.00007 & 0.00011 & 0.00018 & 0.00116 \\
& $\mathrm{~N}_{2}$ & 0.474 & 0.138 & 0.318 & 0.017 & \\
$3 \times 10^{14}$ & $\mathrm{O}_{2}$ & 0.482 & 0.001 & 0.069 & 0.010 & 0.401 \\
& $\mathrm{~F}_{2}$ & 0.372 & 0.001 & 0.111 & 0.052 & 0.208 \\
& $\mathrm{~N}_{2}$ & 0.825 & 0.375 & 0.400 & 0.049 & \\
$5 \times 10^{14}$ & $\mathrm{O}_{2}$ & 0.537 & 0.002 & 0.125 & 0.060 & 0.349 \\
& $\mathrm{~F}_{2}$ & 0.697 & 0.001 & 0.310 & 0.141 & 0.245 \\
\hline \hline
\end{tabular}

We also tabulate the contributions from different types of orbitals. Note that the ionization probability of an individual spin-orbital as in Figs. 1-3 is multiplied by the number of degenerate orbitals to get the total contributions of one type of orbital. These numbers demonstrate that inner valence electrons, such as the $2 \sigma_{u}$ electrons of $\mathrm{N}_{2}$ as well as the $3 \sigma_{g}$ and the $1 \pi_{u}$ electrons of $\mathrm{O}_{2}$ and $\mathrm{F}_{2}$, may contribute substantially to the total ionization. The $\mathrm{N}_{2}, \mathrm{O}_{2}$, and $\mathrm{F}_{2}$ have different ionization dynamics when the inner valence electrons are considered. The inner valence electron ionization is related to the orbital orientation and the enhanced dipole coupling between molecular orbitals at high laser intensities. Multielectron responses of a molecule can also enhance or suppress the ionization of individual electrons. Table II shows in digits that the ionization of the $1 \pi_{g}$ electrons of $\mathrm{O}_{2}$ is suppressed as the laser intensity increases from 3 to 5 $\times 10^{14} \mathrm{~W} / \mathrm{cm}^{2}$. MPIs of $\mathrm{O}_{2}$ are mainly from $1 \pi_{g}$ electrons, therefore multielectron responses may contribute to the observed ionization suppression of $\mathrm{O}_{2}$. The $1 \pi_{g}$ ionization of $\mathrm{F}_{2}$ is not suppressed in a similar manner. To the contrary, multielectron responses of $\mathrm{F}_{2}$ facilitate the ionization of the $3 \sigma_{g}$ electrons whose orbital orientation is parallel to the polarization direction of the laser field. In conclusion, our allelectron calculations reveal electron dynamics that simplified theories cannot model, and demonstrate the importance of multielectron responses originating from the detailed electronic structure for multiphoton ionization of molecules and an array of other molecular multiphoton processes.

This work is supported by the Chemical Sciences, Geosciences, and Biosciences Division of the Office of Basic Energy Sciences, Office of Science, U.S. Department of Energy. We acknowledge the support of the ORIGIN 2400 supercomputer time by the Kansas Center for Advanced Scientific Computing.
[1] K. J. Schafer and K. C. Kulander, Phys. Rev. A 42, 5794 (1990).

[2] H. G. Muller and F. C. Kooiman, Phys. Rev. Lett. 81, 1207 (1998).

[3] A. Becker et al., Phys. Rev. A 64, 023408 (2001).

[4] L. V. Keldysh, Sov. Phys. JETP 20, 1307 (1965).

[5] M. V. Ammosov, Sov. Phys. JETP 64, 1191 (1986).

[6] G. N. Gibson, R. R. Freeman, and T. J. McIlrath, Phys. Rev. Lett. 67, 1230 (1991).

[7] S. L. Chin et al., J. Phys. B 25, L249 (1992).

[8] T. D. G. Walsh, F. A. Ilkov, J. E. Decker, and S. L. Chin, J. Phys. B 27, 3767 (1994).

[9] A. Talebpour, C.-Y. Chien, and S. L. Chin, J. Phys. B 29, L677 (1996).

[10] C. Guo, M. Li, J. P. Nibarger, and G. N. Gibson, Phys. Rev. A 58, R4271 (1998).

[11] M. J. DeWitt, E. Wells, and R. R. Jones, Phys. Rev. Lett. 87, 153001 (2001).

[12] E. Wells, M. J. DeWitt, and R. R. Jones, Phys. Rev. A 66, 013409 (2002).
[13] X. M. Tong, Z. X. Zhao, and C. D. Lin, Phys. Rev. A 66, 033402 (2002)

[14] H. R. Reiss, Phys. Rev. A 22, 1786 (1980).

[15] J. Muth-Bohm, A. Becker, and F. H. M. Faisal, Phys. Rev. Lett. 85, 2280 (2000).

[16] C. Guo, Phys. Rev. Lett. 85, 2276 (2000).

[17] S. J. A. van Gisbergen, F. Kootstra, P. R. T. Schipper, O. V. Gritsenko1, J. G. Snijders, and E. J. Baerends, Phys. Rev. A 57, 2556 (1998).

[18] P. R. T. Schipper, O. V. Gritsenko, S. J. A. van Gisbergen, and E. J. Baerends, J. Chem. Phys. 112, 1344 (1999).

[19] X. Chu and S. I. Chu, Phys. Rev. A 63, 023411 (2001).

[20] X. Chu and S. I. Chu, Phys. Rev. A 63, 013414 (2001).

[21] I. V. Litvinyuk et al., Phys. Rev. Lett. 90, 233003 (2003).

[22] X. Chu and S. Chu (unpublished).

[23] A. Lofthus and P. H. Krupenie, J. Phys. Chem. Ref. Data 6, 113 (1977).

[24] P. Baltzer et al., Phys. Rev. A 45, 4374 (1992).

[25] A. B. Cornford, D. C. Fros, C. A. McDowell, J. L. Ragle, and I. A. Senhouse, J. Chem. Phys. 54, 2651 (1971). 